\title{
LE CATHOLICISME, L'ÉCOLOGIE ET LA MOBILISATION SOCIO-POLITIQUE EN LIGNE AU VIETNAM AUTOUR DE LA CATASTROPHE ENVIRONNEMENTALE « FORMOSA »
}

\author{
Thomas Anh Ngoc HoANG ${ }^{1}$
}

\begin{abstract}
Cette étude propose une analyse communicationnelle du processus de mise en visibilité digitale d'un militantisme catholique vietnamien suite à la catastrophe écologique "Formosa » qui a eu lieu au Centre du Vietnam en avril 2016. Elle s'appuie sur une approche agonistique honnethienne de la reconnaissance sociale et, par conséquent, sur les travaux d'Olivier Voirol portant sur les luttes pour la visibilité. Ainsi, elle montre que cette mise en visibilité a puisé sa force dans l'articulation d'une triple ressource : la religion catholique vietnamienne (doctrine et organisation ecclésiale), un régime axiologique contemporain autour de la question écologique, et une opérativité performante des médias numériques. Contribuant à l'émergence d'un activisme citoyen et d'une société civile dans un Vietnam contemporain sous régime communiste autoritaire, ce militantisme écologique digital des acteurs catholiques vietnamiens permet d'approfondir la « remédiation » conçue comme une quête de « remède ", via le medium numérique, aux préoccupations écologiques et, plus largement, aux problèmes socio-politiques de ce pays vietnamien où cette religion catholique est entre « le ciel et la terre ».
\end{abstract}

1 Thomas Anh Ngoc HoANG est maître de conférences en Sciences de l'Information et de la Communication, membre du Centre Humanités et Sociétés (CHUS) à la Faculté des Humanités à l'Université Catholique de l'Ouest (UCO), Angers, France et également membre associé du laboratoire GRIPIC, Université Sorbonne Paris IV (EA 1498).

Recherches en communication, $\mathrm{n}^{\circ} 53$ - Article publié le 15/09/2021 
Début avril 2016, le rejet en mer de Chine d'une grande quantité de substances toxiques par l'aciérie taïwanaise nommée Formosa Ha Tinh Steel (désormais Formosa) implantée dans le Centre du Vietnam a généré une pollution maritime sans précédent avec au moins 400 tonnes de poissons et crustacés sauvages et d'élevage perdus et près de 450 hectares de corail endommagés ${ }^{2}$. S'étendant sur $200 \mathrm{kms}$ de côte, ce drame écologique a profondément frappé la vie d'une large population vietnamienne vivant notamment de la pêche dans les provinces du Centre du Vietnam. Provoquant ainsi une crise socioéconomique qui touche directement environ 200000 personnes (privation d'emploi de pêcheurs, perte de clientèle du secteur de tourisme, etc.), cette catastrophe écologique a suscité, de par son ampleur, une vive émotion au sein de l'ensemble de la population vietnamienne. Durant trois dimanches de suite, une série de protestations dans la rue se sont produites dans de nombreux endroits au Vietnam pour réclamer plus de transparence sur l'origine de cette pollution et de ses effets. Fin juin 2016, le gouvernement vietnamien reconnaît enfin la responsabilité de la filiale vietnamienne Formosa dans la pollution : celle-ci a présenté ses excuses publiques et a promis de dédommager les victimes avec une somme de 500 millions de dollars ${ }^{3}$. Cependant, même un an après la catastrophe, des manifestations n'ont pas cessé ; elles ont qui plus est fait l'objet de nombreuses violences policières et d'arrestations de la part des autorités publiques.

Dans ce contexte, les paroissiens du diocèse de Vinh, notamment ceux qui se trouvent dans des régions sinistrées, se sont fortement mobilisés, sous la houlette de leurs prêtres, via des manifestations dans les rues, des pétitions auprès des autorités publiques locales et de l'Assemblée Nationale, mais aussi des célébrations de messe et de prière pour la cause de l'environnement, celle du mouvement « Justice et Paix », etc. En mai 2017, Mgr Nguyễn Thái Hợp, évêque du diocèse de Vinh, où habitent environ 600000 catholiques, a conduit une délégation de prêtres pour effectuer une « Marche pour la justice et les droits de l'homme » en Europe avec comme objectif d'alerter l'opinion publique internationale sur la situation des provinces du Centre du Vietnam ${ }^{4}$. En

2 https://www.liberation.fr/planete/2019/06/18/vietnam-apres-la-pollution-formosarattrape-par-la-justice_1734494, consulté le 23 janvier 2020.

3 http://www.europe-solidaire.org/spip.php?article42373, consulté le 23 janvier 2020.

4 https://missionsetrangeres.com/eglises-asie/2017-05-22-ab-nous-attendons-une-reponse-du-gouvernement-et-de-la-societe-formosa-bb-entretien-exclusif-avec-mgrnguyen-thai-hop-eveque-de-vinh/, consulté le 23 janvier 2020. 
août 2017, cette délégation a effectué une autre mission à Taïwan afin de mener une lutte juridique auprès du gouvernement taïwanais ${ }^{5}$. En juillet 2019, une délégation vietnamienne représentants des victimes du désastre maritime de deux diocèses de Vinh et de Hà Tĩnh ont porté plainte contre Formosa à Taïwan ${ }^{6}$.

En parallèle à cette arène de la rue et à celle de la lutte juridique, l'engagement et la mobilisation de l'Église catholique vietnamienne locale pour cette cause environnementale ont également pris la forme d'un militantisme digital. En effet, par le biais des textes, des images, des clips qui sont diffusés notamment sur des sites web catholiques vietnamiens et des réseaux socionumériques (Facebook, YouTube), et repris par la presse de la diaspora vietnamienne et la presse internationale, les acteurs catholiques vietnamiens ont mené un combat important pour contribuer à rendre visibles dans l'espace public les causes écologiques et socio-politiques à travers ce scandale public.

Selon certains analystes non confessionnels (Tran Thi Lien, 2018 ; Hoang Co Dinh, 2017 ; Chung Van Hoang \& Chong, 2017), la grande visibilité médiatique de l'affaire de Formosa, à l'échelle nationale et internationale, qui a fortement « alimenté une crise sociale et politique nationale ${ }^{7} \gg$ au Vietnam depuis 2016, a été rendue possible en grande partie par l'engagement et le militantisme des acteurs catholiques, et, par conséquent, par le prix cher que certains d'entre eux ont dû payer en termes d'emprisonnements, d'agressions, de harcèlement de la part du gouvernement vietnamien ${ }^{8}$.

Ainsi, je propose d'examiner l'affaire de Formosa sous l'angle d'un processus de mise en visibilité digitale par des acteurs catholiques vietnamiens. Il s'agit donc d'interroger ici ce que le religieux fait avec le numérique en supposant le numérique comme une force de mobili-

5 https://dcetvn.org/dgm-phaolo-nguyen-thai-hop-va-ban-van-dong-phap-ly-chonan-nhan-formosa-lam-viec-tai-dai-loan/, consulté le 23 janvier 2020.

6 https://jffv.org/2019/07/14/press-conference-for-indictment-in-taiwan-against-formosa-ha-tinh-steel-corporation-for-ocean-pollution/, consulté le 23 janvier 2020.

7 https://www.acatfrance.fr/actualite/formosa--3-ans-apres---les-defenseurs-de-lenvironnement-toujours-baillonnes, consulté le 23 janvier 2020.

8 Voir l'article sur la $\mathrm{BBC}$ vietnamienne "Người bất đồng chính kiến Công giáo bị "'ngắm»?" [Les dissidents catholiques dans le collimateur ?], en ligne https://www. bbc.com/vietnamese/vietnam-45243420; et l'article sur South China Morning Post "Vietnam's Catholics: cross with China, and all communists", en ligne https:// www.scmp.com/week-asia/politics/article/2160242/vietnams-catholics-cross-china-and-all-communists 
sation en lien avec le domaine religieux. Ce travail se situe alors pleinement à la croisée du nouveau courant de recherche anglophone et allemand des Digital Religion Studies qui « étudient les implications en ligne et hors ligne de la reformulation des pratiques existantes et des nouvelles expressions de spiritualité en ligne » (Campbell, 2017, p. 17) $)^{9}$ et de la perspective francophone religion et numérique en vue d'« une profondeur anthropologique et d'une mise en perspective politique » (Catellani, Douyère \& Servais, 2019). Ainsi, il s'articulera sur deux volets : d'abord, je questionnerai les « tactiques » mises en œuvre par les acteurs catholiques en vue de la mise en visibilité digitale de la « catastrophe de Formosa ». Puis, je chercherai à examiner la place de la dimension écologique mise en avant dans les discours en jeu face à cet événement socio-politique majeur.

Pour ce faire, sur le plan théorique, je m'appuierai essentiellement sur les travaux de la théorie critique d'Axel Honneth concernant la lutte pour la reconnaissance (2013) et ceux d'Olivier Voirol sur les luttes pour la visibilité $(2005 ; 2015)$. Mon pari scientifique consiste à transposer cette théorie critique de l'espace public, conçue initialement pour appréhender la question du conflit social dans le cadre d'un capitalisme occidental avancé, à un tout autre contexte, celui d'une société vietnamienne sous régime communiste autoritaire. Je proposerai donc de déplacer le regard de la théorie normative habermassienne de la communication sociale qui souligne le recours à une raison communicationnelle (Habermas, 1962), fondée sur « l'échange raisonné d'arguments entre des partenaires supposés égaux de discussion » (Carré, 2015, p. 82), vers l'approche agonistique honnethienne de la reconnaissance sociale, afin de penser à frais nouveaux « les termes de la conflictualité sociale en considérant la dimension morale ancrée dans la lutte menée par les acteurs sociaux à partir des expériences négatives qu'ils subissent» (Voirol, 2015, p. 45).

À la lumière de cette approche honnethienne, le scandale public de Formosa pourrait être interprété comme un conflit social où tout un pan de la population vietnamienne, incarné par des catholiques vietnamiens directement ou indirectement concernés par l'affaire de Formosa, s'estime non seulement être victime d'une catastrophe écologique mais surtout avoir fait l'expérience négative de l'injustice sociale et de la répression de la part des autorités vietnamiennes. Ainsi, ils se sont

9 Campbell, « Surveying theoretical approaches within digital religion studies », 2017, p. 17, cité par Andrea Catellani, David Douyère, Olivier Servais, 2019. 
engagés, dans la polis au sens de Hannah Arendt, dans une lutte pour la visibilité, c'est-à-dire « l'agir qui, partant d'un vécu de l'invisibilité ou de la dépréciation symbolique, déploie des procédés pratiques, techniques et communicationnels pour se manifester sur une scène publique et faire reconnaître des pratiques ou des orientations politiques " (Voirol, 2005, pp. 107-108).

Mon analyse se focalisera sur des médias numériques. Dans cette « scène publique » conçue comme un lieu d'apparition médiatique, j'examinerai une lutte pour une visibilité médiatique digitale menée par ces acteurs marginaux qui constitueraient un « espace public oppositionnel» (Negt, 2007), défini comme la rencontre de toutes « les subjectivités rebelles ", donnant naissance à des formes d'opposition et ayant des visées d'émancipation sociale dans le contexte particulier d'une société vietnamienne sous un régime autoritaire.

Mon corpus est constitué essentiellement de sources médiatiques digitales issues des acteurs catholiques vietnamiens nationaux et d'outre-mer, des acteurs catholiques internationaux, mais aussi des acteurs médiatiques non catholiques qui participent à la mise en visibilité digitale de cette lutte aux enjeux multiples (écologiques, sociaux, politiques, spirituels et religieux). Il s'agit donc de montrer les visions de ces acteurs qui cherchent à "faire voir et faire valoir ce qui, en quelque sorte, est déjà "sous nos yeux" mais ne peut être thématisé selon les schèmes d'intelligibilité disponibles », par conséquent « indépendamment des discours institués dans l'espace public dominant » (Voirol, 2005, pp. 115-116).

Concrètement, en langue vietnamienne, mon attention se focalisera notamment sur le site web « Tin Mừng Cho Người Nghèo »[littéralement " la Bonne Nouvelle pour les Pauvres », souvent abrégé en anglais « GNsP » pour " Good News to the Poor »] https://www. tinmungchonguoingheo.com qui a publié, selon mon recensement, 213 articles sur l'affaire de Formosa entre le 24 avril 2016 et le 8 décembre 2019. Ce site web est accompagné par la page Facebook correspondante éponyme https://www.facebook.com/tinmungchonguoingheo/ et d'une chaine YouTube intitulée "AmenTV » https://www.youtube.com/c/AMENTvVN. Par ailleurs, je prendrai en compte d'autres médias catholiques vietnamiens tels que le site web du diocèse de Vinh http://giaophanvinh.net/, celui de la Conférence des évêques vietnamiens https://www.hdgmvietnam.com, etc.

Ce processus de mise en visibilité digitale a aussi été rendu possible et amplifié par d'autres acteurs médiatiques qui visent notamment 
un public vietnamophone d'outre-mer. En français, on constate de nombreux articles publiés par EDA (Églises d'Asie) - l'agence d'information des Missions Étrangères de Paris -, quelques publications du quotidien la Croix et d'autres sites de médias francophones.

Sur le plan méthodologique, pour l'analyse des médias numériques j'adopte une approche sémiotique pour articuler différentes dimensions constitutives de ces dispositifs : dimension matérielle et technique, dimension symbolique des langages, et dimension sociale des pratiques de communication (écriture) (Souchier \& Jeanneret, 2009). En plus de cette sémiotique appliquée à l'écrit d'écran, je pratiquerai la méthode de l'analyse de contenu notamment à deux endroits majeurs : d'abord, le choix des articles pertinents sur le site web de « $\mathrm{GNsP}^{10} »$ concernant l'affaire Formosa (213 articles); puis, une classification thématique de ces 213 articles en cinq grands thèmes ${ }^{11}$ (voir l'analyse infra partie 2).

Avant de poursuivre, il m'est important d'expliciter ma position en tant que chercheur par rapport à cette étude. Étant d'origine vietnamienne et de confession catholique, j'assume pleinement la part d'implication et de proximité que j'ai avec le " terrain » étudié. Je suis conscient que "l'écriture de soi " est à l'œuvre dans mon travail de chercheur, mais que " me faire apparaître par moments dans mon texte, choisir un $j e$ qui dit d'où $\mathrm{j}$ 'observe est un gage scientifique parmi d'autres » (Lambert, 2007, pp. 35, 39). Ainsi l'éclairage épistémologique que proposent Daniel Cefaï et Valérie Amiraux (2002) sur la dimension de «l'engagement de soi » en sciences sociales me paraît pertinent :

« La compréhension d'un objet d'enquête ne se réduit pas, en effet, à l'ensemble des connaissances scientifiques ou des données factuelles qui ont pu être rassemblées à son propos. Le « flair » du chercheur, qui l'amène à voir des configurations de sens que d'autres ont ignorées ou à proposer des lectures hors des routines d'une discipline, n'est

10 J'ai effectué la recherche avec le mot clé «formosa » à partir de la rubrique « Recherche » de ce site web. Puis, j'ai vérifié l'adéquation de chaque article avec la thématique de «l'affaire de Formosa » sur la base d'une lecture rapide.

11 Ici je procède par une démarche habituelle de l'analyse de contenu de ces articles : lecture, classification, interprétation. Ce travail laborieux (entre autres, une lecture attentive de 213 articles) me permettra d'identifier des passages particulièrement significatifs que je citerai dans mon analyse. 
souvent rien d'autre que sa capacité pratique à user du raisonnement analogique, ou, plus largement, à recourir à des schèmes de perception et d'action, d'orientation et de jugement qu'il a incorporés au cours de sa trajectoire biographique ».

Ma maitrise de la langue vietnamienne (langue maternelle), ma connaissance approfondie du contexte vietnamien, mes propres expériences de « vietnamien catholique » seraient ainsi autant d'éléments favorables à mon investigation. En même temps, je me dois aussi de prendre « une position d'impartialité négociée ${ }^{12} »$, car «c'est avec et contre soi qu'il faut se battre pour produire du savoir » (Cefaï \& Amiraux, 2002). Par conséquent, en vue d'une investigation objectivante et distanciée, j'aurai recours essentiellement à un cadre théorique nonconfessionnel (théorie critique de Honneth, de Voirol) et je convoquerai des sources plurielles et diverses : non vietnamiennes et vietnamiennes, catholiques et non catholiques.

\section{La mise en visibilité digitale de la « catastrophe de Formosa » par les acteurs catholiques vietnamiens}

Ici, l'analyse porte sur les «manières de faire » des acteurs catholiques vietnamiens pour produire leur propre discours et donc façonner une certaine « vision catholique » de l'affaire Formosa.

Le cas du site web « la Bonne Nouvelle pour les Pauvres » (désormais « GNsP ») est éclairant. Sur le plan de l'énonciation, le site affiche clairement une position de l'engagement catholique explicite envers les « pauvres » dans sa présentation: " Nous annonçons la Bonne Nouvelle aux plus abandonnés et portons la voix des personnes victimes de l'injustice, des sans-voix ${ }^{13}$. » Le parti pris de ce média se traduit par la présence, outre les thématiques habituelles d'un site religieux (homélie, lectures, documents catholiques, etc.), de la rubrique " Dân oan » [Population victime d'injustice sociale] et « Phóng sự » [Reportages] qui se focalisent sur des problèmes socio-politiques de la société vietnamienne contemporaine. Probablement créé en 2015, le site semble

12 Selon l'expression de Jürgen Habermas, Morale et communication, Paris, Cerf, 1986, pp. 41-54, cité par Lambert, op. cit. p. 15.

13 https://www.tinmungchonguoingheo.com/about/ consulté le 23 janvier 2020. 
très visité ${ }^{14}$. Il présente des liens vers des sites catholiques institutionnels (Vatican, conférence des évêques du Vietnam, commission Justice et Paix, les grands diocèses vietnamiens), mais aussi vers des médias internationaux non confessionnels en langue vietnamienne et le portail d'information catholique vietnamien d'outre-mer Vietcatholics, qui sont censés échapper à la propagande de l'État-Parti vietnamien. Ces éléments d'énonciation permettent donc de saisir une « ligne éditoriale » de ce média : au nom de la foi catholique, il se situe pour la cause des " pauvres », des « victimes de l'injustice sociale», en dehors du contrôle du gouvernement vietnamien. Ainsi ce média s'inscrit pleinement dans cette perspective du conflit social élaboré dans l'approche agonistique honnethienne de la reconnaissance sociale, «à l'aune des motifs moraux, du vécu de l'injustice et des atteintes à la dignité » (Voirol, 2015, p. 49). Il atteste également de la pertinence de la théorie de luttes pour la visibilité de Voirol car " des pans entiers de l'expérience sociale demeurent dans l'ombre et le silence, condamnant dès lors des situations, des expériences, des acteurs et des pratiques à rester en marge de l'attention publique » (Voirol, 2005, p. 99).

Pour comprendre le fonctionnement de ce média catholique au sein du paysage médiatique fortement sous contrôle, on peut convoquer la notion de « tactique » de Michel de Certeau dans un rapport de forces :

« J'appelle tactique l'action calculée que détermine l'absence d'un propre. [...]. La tactique n'a pour lieu que celui de l'autre. Aussi doit-elle jouer avec le terrain qui lui est imposé tel que l'organise la loi d'une force étrangère. [...] elle est mouvement "à l'intérieur du champ de vision de l'ennemi" [...], et dans l'espace contrôlé par lui » (Certeau, 1990, pp. 60-61).

Dépourvu d' « un lieu propre », le site web GNsP doit « jouer avec» le « terrain imposé » et « l'espace contrôlé » par le régime au pouvoir. En effet, hébergé hors du Vietnam ${ }^{15}$, le média fonctionne avec la collaboration des « journalistes GNsP », comme on peut le constater dans la

14 Il comptabilise 3006105 visites le 20 janvier 2020 .

15 C'est la pratique courante des sites web alternatifs pour éviter ou réduire des attaques des forces de cybersécurité du gouvernement vietnamien. En plus, le téléphone de contact affiché pour la page Facebook de « GNsP » indique un numéro situé aux États-Unis. 
signature des articles de ce site qui couvraient des manifestations lors de l'affaire de Formosa. En s'appuyant sur l'investigation de Nguyen Thanh Giang concernant le fonctionnement du portail Vietcatholics News (Nguyen, 2010, p. 10), on pourrait déduire que ces « journalistes GNsP » seraient des activistes catholiques vietnamiens qui produisent leur information à partir de leur « terrain ». La spécificité de ce média catholique qui fait sa force réside donc dans ce réseau souple, informel et « souterrain » de collaborateurs, qui sont des fidèles catholiques installés sur l'ensemble du pays et qui contribuent à produire des informations conformément à leur vision religieuse.

À titre d'exemple, l'article intitulé « Reportage sur des manifestations dans tout le pays contre la grave pollution maritime ${ }^{16}$ » donne à voir des photos prises lors des manifestations dans de nombreuses villes du Vietnam, ce qui suppose la contribution de plusieurs « journalistes GNsP » à cet article. L'aspect non professionnel de ces photos montre un caractère amateur des auteurs, mais atteste de la " véracité " de ce qui se montre. Cette volonté de «monstration" par le visuel de GNsP relève de l'intention de "dire la vérité » en opposition et en résistance aux médias officiels mainstream perçus comme soumis à la seule visée propagandiste de l'État-Parti vietnamien (Abuza, 2015) : les images fonctionnent comme des preuves de « vérité », car les « informateurs » sont aussi des « témoins » qui exposent leurs propres expériences et celles de leurs confrères.

Ici, la modalité de témoignages rendus visibles dans ce média catholique permet, dans la perspective honnethienne, de reconstruire une morale dominée, par " une déconstruction de la surface la plus apparente de la communication sociale de l'espace public majoritaire » (Honneth, 2013). Il importe donc de souligner son intérêt méthodologique : une attention phénoménologique aux modalités pratiques de l'expérience négative de l'injustice subie par une catégorie de la population marginale.

En raison des « schèmes d'intelligibilité » de ce média catholique « en rupture avec les modalités dominantes de la mise en récit» (Voirol, 2005, p. 115) des médias officiels vietnamiens sous contrôle, certains articles du site GNsP sur l'affaire de Formosa ont été repris, cités par

$16 \mathrm{https} / /$ www.tinmungchonguoingheo.com/blog/2016/05/01/tuong-thuat-ca-nuocbieu-tinh-vi-bien-bi-o-nhiem-nghiem-trong-xin-nhan-f5/, consulté le 23 janvier 2020 . 
d'autres médias non confessionnels, comme la $\mathrm{BBC}$ vietnamienne ${ }^{17}$, ou le blog « carnet de recherches » Mémoires d'Indochine de l'universitaire français François Guillemot ${ }^{18}$ (Figure 1).

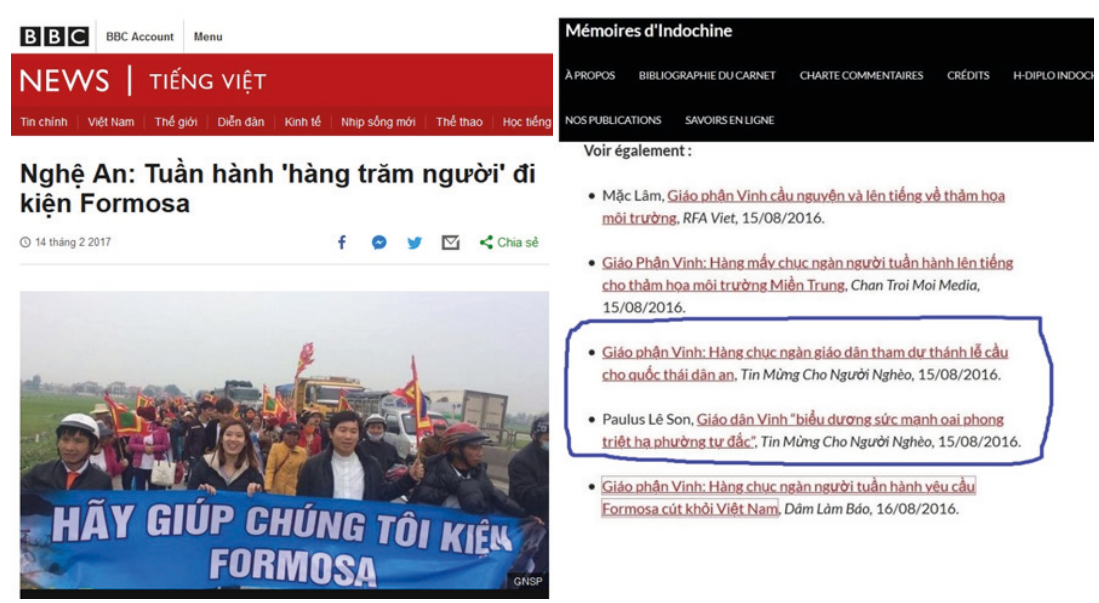

Figure 1. Reprise de la source d'informations du média GNsP par la BBC vietnamienne et le blog Mémoires d'Indochine

Par ailleurs, une autre « force de frappe » de ce média catholique vietnamien réside dans sa page Facebook et sa chaîne YouTube qui font partie de l'ensemble du dispositif numérique qui a œuvré pour la mise en visibilité digitale de l'affaire Formosa (voir Figure 2). Au Vietnam, les médias sociaux constituent aujourd'hui une « arène politique » (Bui, 2016, p. 97), sachant que Facebook et YouTube sont largement utilisés par la population vietnamienne ${ }^{19}$.

Au-delà du site web GNsP, la visibilité digitale de l'affaire de Formosa a été rendue possible par d'autres acteurs médiatiques catholiques vietnamiens, tels que le site web du diocèse de Vinh publiant un communiqué de la Commission « Justice \& Paix » dès le début du désastre, ou le site web de l'épiscopat vietnamien qui exprime, par leur Lettre commune de 2016, leur solidarité avec les habitants sinistrés et appeler

17 https://www.bbc.com/vietnamese/vietnam-38955098, consulté le 23 janvier 2020.

$18 \mathrm{https}: / /$ indomemoires.hypotheses.org/tag/diocese-de-vinh, consulté le 23 janvier 2020.

19 Le nombre d'utilisateurs de Facebook devait atteindre 45,3 millions en 2019 sur un total de population de 95,5 millions, voir https://www.statista.com/statistics/490478/ number-of-vietnam-facebook-users/, dernière consultation le 23 janvier 2020. 
le gouvernement à " être à l'écoute des aspirations légitimes de son peuple ${ }^{20} »$.

Outre ces dispositifs numériques, les acteurs catholiques vietnamiens s'appuient sur la force d'une structure ecclésiale très organisée et solidement ancrée dans le catholicisme vietnamien de par sa longue histoire. En effet, avant tout, de nombreux fidèles catholiques des régions affectées par le désastre se sont fortement mobilisés pendant des mois dans des manifestations pour réclamer de la transparence sur la catastrophe ou pour demander une juste indemnisation, dans des marches pacifiques pour l'environnement, des vigiles de prières et des messes pour la « justice et paix », etc. Des responsables religieux comme certains prêtres locaux et certains évêques se sont aussi très largement engagés dans cette lutte écologique et politique.

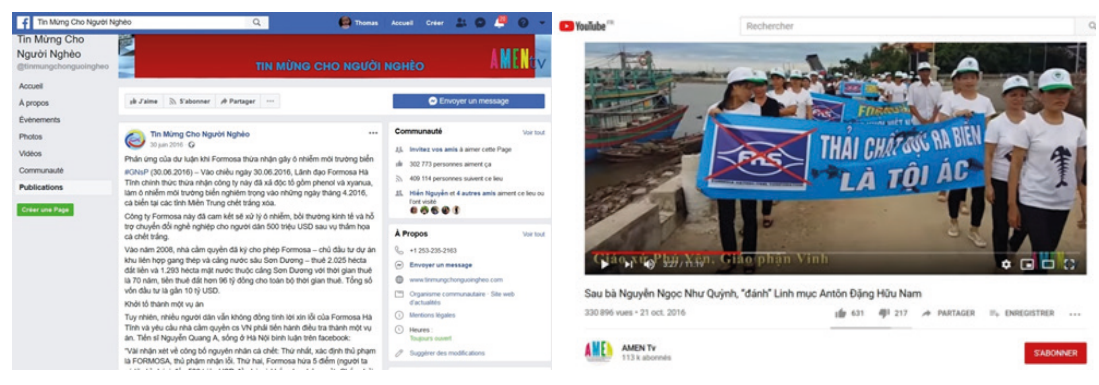

Figure 2A. La page Facebook "Tin Figure 2B. Chaîne Youtube « AmenMừng cho Người Nghèo » GNsP por- TV » portant sur l'affaire Formosa tant sur l'affaire Formosa

Par ailleurs, historiquement, la communauté catholique vietnamienne s'est trouvée contrainte de «vivre avec » le régime communiste depuis 1954 au Nord-Vietnam, et depuis 1975 au Sud-Vietnam (Tran Thi Lien, 2010, pp. 347-348). Cette expérience lui a certainement servi dans cette lutte contemporaine. Ce «catholicisme à l'ancienne » des Vietnamiens (Passicousset \& Papin, 2010, p. 291) où les fidèles font confiance et obéissent à leurs responsables (prêtres, évêques), où ces « fidèles très pratiquants, généreux et organisés » font communauté « dans une société filiale, fondée sur le culte des ancêtres et des

20 La Lettre commune en vietnamien : https://www.hdgmvietnam.com/chi-tiet/thuchung-2016-31644 consulté le 23 janvier 2020. 
morts, sur le respect des anciens ${ }^{21} »$, constitue « une sorte de môle de résistance » (Passicousset \& Papin, op. cit., p. 290) pour résister aux stratégies (médiatiques, policières, etc.) du pouvoir public.

Enfin, étant une religion « universelle », le catholicisme vietnamien puise sa force aussi dans le réseau catholique global, notamment dans des médias catholiques internationaux comme le site web Églises d'Asie des Missions étrangères de Paris, le journal français La Croix, etc. En traduisant en français des textes des acteurs catholiques vietnamiens (communiqué, lettre commune, etc.), ou en relayant les manifestations des acteurs catholiques vietnamiens, le site web Églises d'Asie des MEP a contribué à porter sur la scène internationale la visibilité digitale et la visibilité tout court de cet activisme catholique vietnamien.

Ainsi, il importe de noter, dans le sillage de l'approche de Honneth sur la « liberté sociale » et l'ambivalence de l'espace public (Honneth, Le droit de la liberté, 2015, cité par Carré, 2015), les potentiels de 1' « émancipation sociale» des dispositifs numériques. En effet, les « contre-publics » catholiques vietnamiens en ligne - les « publics faibles » selon la terminologie de Fraser (2005) -, en s'appuyant sur des structures et des publics catholiques vietnamiens et internationaux hors ligne, les fortifient et leur confèrent une visibilité digitale dans la sphère publique médiatique.

À cette étape, l'analyse a mis en lumière les « manières de faire » et les tactiques des acteurs catholiques vietnamiens afin de lutter pour la visibilité de leurs propres discours et de leur propre vision dans l'arène médiatique numérique alternative d'aujourd'hui. Il s'agit maintenant d'approfondir la problématique écologique qui est en effet au cœur de cet activisme religieux.

\section{De la lutte écologique à une prise de conscience politique généralisée par la population vietnamienne d'aujourd'hui}

Une analyse de contenu effectuée à partir des 213 articles du site web GNsP a permis d'identifier les cinq grandes thématiques dont deux se focalisent explicitement sur la question écologique (Figure 3) : les « dégâts de la catastrophe Formosa » $(6 \%)$ et « l'engagement pour la cause écologique » $(16 \%)$, ou indirectement : « engagement des acteurs catholiques » (aussi pour la cause écologique) $(24 \%)$ et « récits de

$21 \mathrm{https}: / /$ missionsetrangeres.com/eglises-asie/leglise-vietnamienne-une-eglise-familiale/ consulté le 23 janvier 2020. 
manifestation » $(20 \%)$ (en raison des problèmes écologiques). Donc, le fil rouge de la mise en discours de ce média catholique est essentiellement la question écologique. L'autre thématique, qui reste, porte sur « la critique du pouvoir public » (34\%), qui découle comme la conséquence logique de cette conscience écologique « activée » à l'occasion de ce scandale écolo-politique.

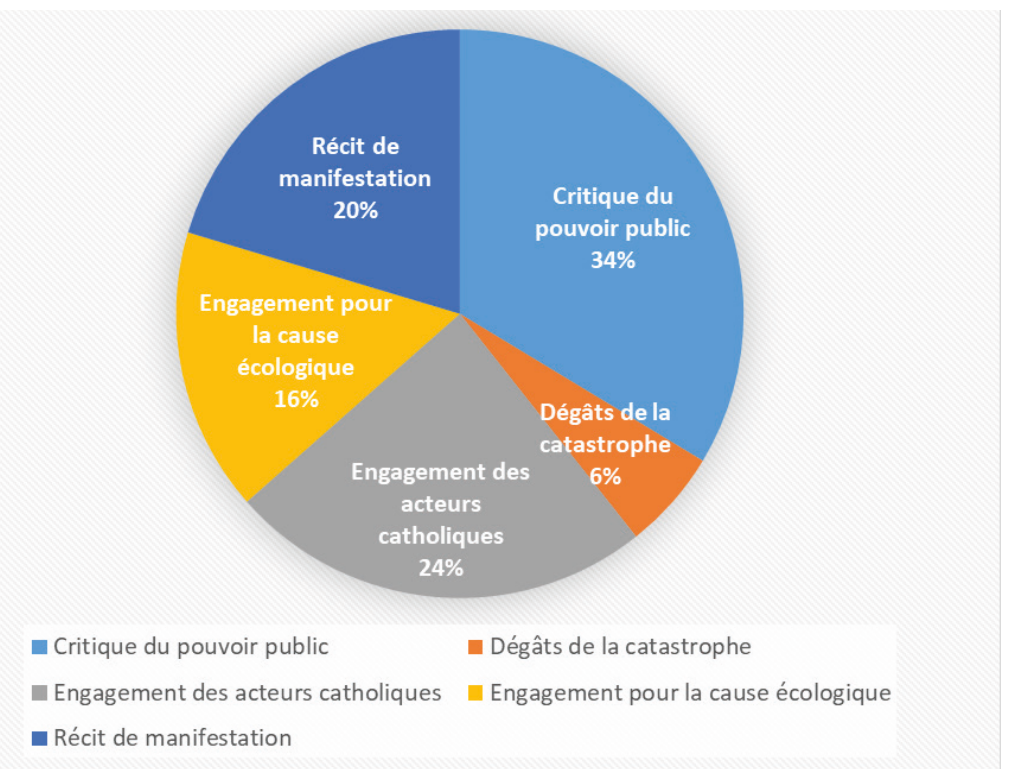

Figure 3. Grandes thématiques à partir des articles sur Formosa du site web «GNSP 》

Le site met en scène des dégâts de la catastrophe en montrant souvent des photos de poissons morts (conséquences sur la nature) et en mettant en récit les souffrances des victimes (conséquences sur les personnes humaines). Une des photos qui circule beaucoup sur des supports médiatiques donne à voir un pêcheur vietnamien montrant, dans un geste " démonstratif ", des poissons morts. Elle est utilisée aussi bien par un média officiel (gauche) que par un livre à visée critique, coordonné par un auteur vietnamien d'outre-mer (droite) (Figure 4). Porteuses de la force d'un logos persuasif par la figure d'exemple (une preuve palpable), d'un ethos de solidarité et de vérité, ces photos circulantes de poissons morts suscitent aussi de fortes émotions, un pathos dramatique et compassionnel, ce qui construit la performance communicative de la cause écologique. Quant aux récits évoquant les lourds 
dégâts de la pollution maritime sur la vie des habitants dès le début du désastre, voici un exemple :
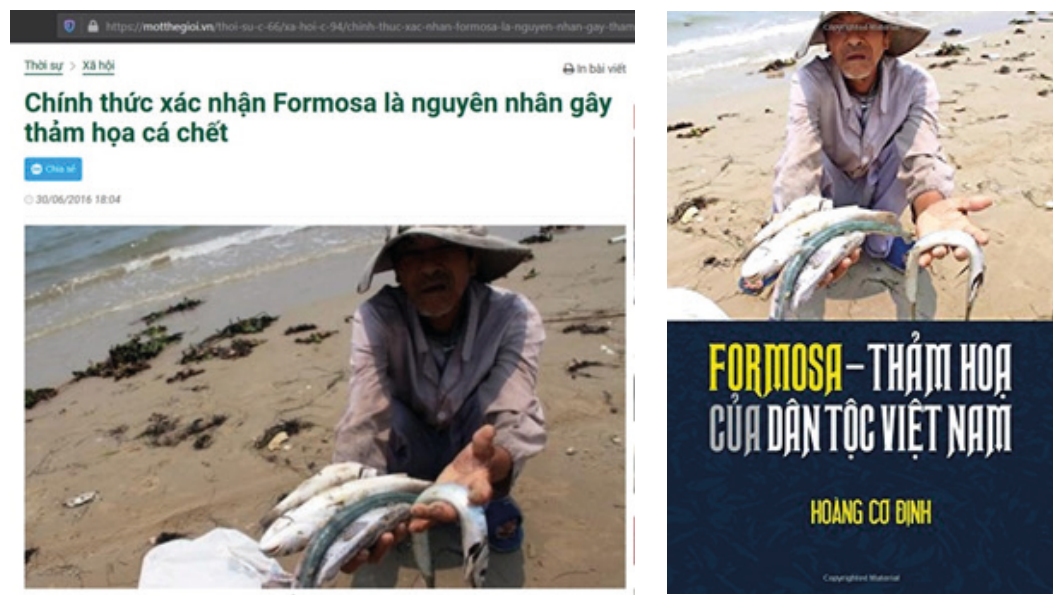

Figure 4. Photo circulant sur différents supports qui montre un pêcheur présentant des poissons morts

« Un certain nombre d'habitants vivant aux alentours de la côte de Vũng Án nous confient que la population est en colère et qu'ils ne savent plus où leur vie va, car ils ne savent quoi faire, à part la pêche. Ils disent que depuis plus d'un mois ils n'ont aucune ressource autre. Si cette situation continue, ils vont mourir ${ }^{22}$. »

Mettant en danger la vie de nombreux habitants, le désastre Formosa soulève une question plus globale sur la crise écologique actuelle $\mathrm{au}$ Vietnam. D'une manière réflexive et critique, une enseignante (Trần Thị Lam) de la province Ha Tinh a composé un poème intitulé « Đất nước mình ngộ quá phải không anh ? » [Notre pays est vraiment étrange, n'est-ce pas ?] qui lance une interpellation pressante et douloureuse face à un sombre avenir du Vietnam suite à ce désastre écologique. Circulant rapidement et largement en ligne, ce poème a été mis en musique par de nombreux Vietnamiens, et circule fortement sur YouTube.

22 « Perte d'emploi et les manifestations de pêcheurs ", https://www.tinmungchonguoingheo.com/blog/2016/04/28/mat-nghiep-va-cac-cuoc-bieu-tinh-cua-ngu-dan/. consulté le 23 janvier 2020. 
En montrant cette situation préoccupante de l'environnement vietnamien actuel, le site web GNsP met en lumière l'engagement des acteurs catholiques pour la cause écologique. Par exemple, une « Journée pour l'Environnement » a été organisée le 7 aout 2016 à l'initiative de la Commission " Justice \& Paix » du diocèse de Vinh « face à la catastrophe écologique qui a lieu actuellement dans les provinces du Centre du Vietnam et en réponse à l'appel du Saint-Père François dans l'encyclique Laudato sí pour élever la conscience de protection de l'environnement au sein de la communauté23 ». L'article montre donc clairement que le fondement doctrinal de l'Église catholique est à l'origine même de cette pratique pastorale : protéger l'environnement, c'est désormais un devoir des catholiques.

L'universitaire Tran Thi Lien Claire explique le contexte :

«Alors que le pays est confronté aux conséquences écologiques d'un développement industriel à marche forcée et du changement climatique, l'Église du Vietnam a été particulièrement sensible à l'encyclique Laudato sí du pape François. [...] L'encyclique a été reprise dans tous les diocèses et la quête du Vendredi Saint est désormais dans tout le Vietnam destinée à l'aide en urgence aux populations victimes des désastres naturels » (Tran Thi Lien, 2018).

Pour une hauteur de vue, il semble pertinent de situer cette communication environnementale de crise sur le scandale de Formosa portée par les catholiques vietnamiens dans le cadre plus global de l'Église catholique, à la fois « universelle» et locale (vietnamienne). En effet, sur le plan doctrinal, l'encyclique Laudato sí, avec le sous-titre «Sur la sauvegarde de la maison commune ", qui est pour la première fois un texte de ce niveau magistériel entièrement consacré au thème écologique et qui a reçu un écho très favorable bien au-delà de la sphère catholique, n'est-il pas un signe que « L'écologie, (est) nouvel horizon de l'Église ${ }^{24} »$ pour notre temps ?

Au niveau local de l'Église catholique vietnamienne, le souci de la protection environnementale apparaît déjà plus tôt dans la « Lettre pas-

23 https:/www.tinmungchonguoingheo.com/blog/2016/07/28/giao-phan-vinh-tochuc-ngay-bao-ve-moi-truong/ consulté le 23 janvier 2020.

24 https://www.la-croix.com/Religion/Catholicisme/Monde/Lecologie-nouvel-horizon-lEglise-2019-09-16-1201047874, consulté le 23 janvier 2020. 
torale » de 2009 de l'archevêque de Saigon qui soulignait : «L'environnement naturel est un don du Créateur que nous pouvons tous partager mais que nous devons surtout préserver pour les générations à venir [...] Protéger l'environnement est le devoir du chrétien ${ }^{25}$. „ Dans leur « Lettre commune adressée au peuple de Dieu » de 2015, la Conférence des évêques vietnamiens a appelé les fidèles vietnamiens à « se convertir, c'est-à-dire changer ses manières de voir, de penser et de vivre dans ses relations avec la nature [...] en commençant par des petits gestes au quotidien ${ }^{26} \gg$.

Vue sous cette large optique, la forte mobilisation, en ligne et hors ligne, des fidèles et des responsables catholiques vietnamiens dans ce scandale de Formosa, au-delà de la revendication des droits légitimes au dédommagement juste pour les victimes, s'inscrit donc pleinement dans une cause délibérément liée à l'écologie intégrale, qui amène à dénoncer « la nature systémique de la crise écologique lié au modèle de développement adopté » (Tran Thi Lien, op. cit.) : en effet, selon le pape François « tout est lié » (Laudato sí, n 70, 91), « il n'y a pas deux crises séparées, l'une environnementale et l'autre sociale, mais une seule et complexe crise socio-environnementale » (Laudato sí n ${ }^{\circ} 139$ ).

Ainsi, à la lumière de cette écologie intégrale, la prise de conscience écologique des catholiques vietnamiens les a amenés à critiquer le (éco) système du régime communiste au pouvoir perçu comme responsable de cette crise écologique. En effet, l'instance ecclésiale la plus élevée, la Conférence des évêques du Vietnam, a livré, dans sa « Lettre commune adressée au peuple de Dieu » de 2016, une analyse ouvertement critique en pointant du doigt le caractère systémique des graves problèmes que traverse le Vietnamien actuel (corruption, catastrophes écologiques, sécurité alimentaire) ainsi que les « causes objectives et de niveau macro qui ont abouti à cette triste réalité ».

Quant à la vision du " peuple », dans un article intitulé " La mort de l'âme ", un auteur du GNsP écrit, avec des métaphores fortes : « Aujourd'hui, les Vietnamiens prennent leur repas avec plein d'incertitude, incapables de savoir quelle nourriture est propre, et quelle nourriture est sale, la frontière entre l'un et l'autre étant si minime.

25 L'intégralité de cette Lettre en vietnamien : https:/tgpsaigon.net/bai-viet/thu-muctu-ve-bao-ve-moi-truong-58340, consulté le 23 janvier 2020.

26 L'intégralité de cette Lettre en vietnamien : https://www.hdgmvietnam.com/chi-tiet/ thu-muc-vu-2015-mo-nam-thanh-long-chua-thuong-xot-31659, consulté le 23 janvier 2020 . 
[...] Le sang toxique dans l'âme est le fruit amer d'une éducation d'un régime totalitaire du Parti communiste athée pendant plus de 70 ans au Nord et plus de 40 au $\mathrm{Sud}^{27}$. » En opérant un glissement sémantique des aliments insalubres vers le «fruit toxique » du système éducatif vietnamien placé sous régime communiste, l'auteur s'inscrit pleinement dans cette conscience de "l'écologie intégrale » pour dénoncer une crise profonde écolo-socio-politique de la société vietnamienne actuelle. Ainsi, cette lutte écologique des catholiques vietnamiens a fini par prendre une dimension politique, au sens étymologique d'une action ordonnée pour le bien commun de la Cité.

De cette manière, la lutte écologique et politique des acteurs catholiques vietnamiens a été rejointe par les militants non confessionnels (militants écologiques - les ONG, les militants politiques - dissidents luttant pour les droits de l'homme, etc.). En témoigne la pétition en ligne (https://thamhoaformosa.com/fr/) lancée un an après l'éclatement du désastre et qui implique cette pluralité d'acteurs unis autour de la cause commune de justice pour les victimes de Formosa. Ainsi, ce militantisme écologique, qui a réussi à établir un pont entre divers acteurs multiples et pluriels autour de Formosa a suscité une prise de conscience citoyenne, une société civile émergente au sein d'une société vietnamienne sous régime communiste (Nguyen \& Datzberger, 2018, p. 10).

Cette analyse met en lumière donc le caractère heuristique de la théorie critique de l'espace public de Honneth, en la déplaçant du cadre initial du capitalisme avancé vers celui d'une société sous régime totalitaire. Parmi les trois facteurs explicatifs de la constitution de l'espace public dans la société moderne selon Honneth, outre le facteur technologique et le facteur culturel, c'est le facteur juridico-politique qui constitue des enjeux de lutte majeurs au Vietnam contemporain en termes de droits politiques réels des citoyens, notamment la liberté d'expression.

\section{Conclusion}

Dans leur livre intitulé « Vivre avec les Vietnamiens », les auteurs français Philippe Papin et Laurent Passicousset posent la question suivante : au Vietnam, «Religions : le ciel ou la terre ?» (2010, p. 287). Cette étude montre qu'au moins pour le catholicisme vietnamien, la religion est « entre la terre et le ciel ». Au commencement de l'af-

27 https://www.tinmungchonguoingheo.com/blog/2016/06/22/cai-chet-cua-tam-hon/, consulté le 23 janvier 2020. C'est moi qui souligne. 
faire Formosa, c'était une mer polluée, des poissons morts, puis des pécheurs en perte d'emploi, c'est-à-dire une mer qui ne donne plus la vie. Donc cette vie « terrestre », bel et bien de la terre, qui est menacée, a inquiété les habitants, dont beaucoup sont des catholiques, et les a conduits à descendre dans la rue pour rendre audible leur voix, visible leur angoisse existentielle. Puis, ces manifestations de rue ont trouvé un autre « lieu » pour leur apparition publique : les médias numériques qui « visibilisent », amplifient mais aussi métamorphosent leur combat pour la vie, mais également pour la justice, la dignité.

En vietnamien, le pays se dit « đất nước », littéralement « terre eau ». Pour eux, la nature, les matières premières, «terre » et « eau » c'est la vie physique, mais aussi la vie symbolique, commune, sociale. Le désastre de Formosa leur a permis de prendre conscience de ce lien vital, intrinsèque de ce double niveau de la vie, physique et symbolique. Cela entre en résonance avec la vision catholique de l' « écologie intégrale », traduite dans une approche anthropologique de la relation, telle qu'exprime le leitmotiv « tout est lié » de l'encyclique Laudato si du pape François.

Ainsi dans cette perspective de liens, cette investigation peut-elle se lire aussi comme un effort d'examiner l'enchevêtrement de trois dimensions communicatives autour du scandale public de Formosa :

- La communication religieuse met en lumière les ressources du catholicisme vietnamien, à la fois en termes de foi (une lutte au nom de la "vérité », de la " justice », clairement exprimée dans le fondement doctrinal tel que Laudato si) et d'organisation ecclésiale (une lutte organisée fondée sur une structure ecclésiale solidement charpentée articulant des forces internes et externes, locales et globales) ;

- La communication écologique donne à voir les enjeux d'une prise de conscience écologique généralisée de la population vietnamienne face aux préoccupations environnementales actuelles de son pays ;

- La communication de crise permet de voir, dans une lutte pour la visibilité et pour la reconnaissance menée par des victimes de l'affaire de Formosa à partir de leur vécu de l'oppression, l'émergence d'un espace social critique commun, constitué d'acteurs multiples et hétérogènes. 
Ici, l'approche critique de l'espace public de Honneth et la théorie de la lutte pour la visibilité de Voirol se sont avérées très pertinentes pour le cas des sociétés sous régime totalitaire comme le Vietnam. Un intérêt méthodologique particulier consiste ici dans une attention phénoménologique aux expériences négatives subies par une catégorie de population marginale.

Ces trois dimensions communicatives s'appuient sur le recours aux médias numériques qui leur permet une visibilité performante. Cette mise en visibilité digitale ne participerait-elle pas d'une logique de « remédiation » qui signifie alors, non seulement le recours à d'autres formes médiatiques plus récentes (numériques), mais aussi, dans cette " mise en abîme ", une quête de " remède », via le medium numérique (sens du latin remedium) ${ }^{28}$, aux préoccupations socio-environnementales du Vietnam contemporain?

En conclusion, il me semble bon de concevoir le religieux, comme le propose Michel Serres dans son dernier ouvrage, à partir de sa double étymologie latine religere et religare, comme le geste de "relire le relié »: " il relie, selon un axe vertical, le ciel et la terre, et, horizontalement, les hommes entre eux » (Serres, 2019, 4e page de couverture). La mise en visibilité catholique digitale vietnamienne du désastre écologique de Formosa n'est-elle pas une manière de " relire » la question écologique, c'est-à-dire celle de s'occuper de notre « maison commune » (oikos) en proposant " liaisons et relations », " une sorte d'ouverture à la synthèse ", afin qu'elle " nous conduise à poser ces questions intégrales aux réponses indécidables »? «Elle nous entraîne à visiter ou à construire le monde dans lequel nous habitons, indissolublement naturel et humain ; en pratique, elle façonne notre maison » (Serres, 2019, p. 218).

\section{Références}

Abuza, Z. (2015). Stifling the Public Sphere: Media and Civil Society in Vietnam. National Endowment for Democracy, Washington, DC. Disponible à : https://www. ned.org/stifling-the-public-sphere-media-and-civil-society-in-egypt-russia-andvietnam/

28 Je m'appuie sur la discussion suivante : « Compte rendu de la séance "Remédiation / mise en abîme" ", Jean Klucinskas, novembre 2003, http://archee.qc.ca/ar.php?page $=$ imp\&no=216, consulté le 23 janvier 2020. 
Bui, T. H. (2016). The Influence of Social Media in Vietnam's Elite Politics. Journal of Current Southeast Asian Affairs 35(2), 89-111. Disponible à : https://nbn-resolving. org/urn:nbn:de:gbv:18-4-9554

Campbell, H. (2017). Surveying Theoretical Approaches within Digital Religion Studies. New Media \& Society, 19(1), 15-24. Doi : https://doi.org/10.1177/1461444816649912

Catellani, A., Douyère, D. \& Servais, O. (2019). Visibilités digitales du religieux, appel à contribution pour la revue Recherches en Communication.

Carré, L. (2015). Le public et ses évolutions manquées. La contribution d'Axel Honneth à une théorie critique de l'espace public. Réseaux 193(5), 79-104. Doi : https:// doi.org/10.3917/res.193.0079

Cefaï, D. \& Amiraux, V. (2002). Les risques du métier. Engagements problématiques en sciences sociales. Partie 1. Cultures \& Conflits [En ligne], 47(3) | automne, mis en ligne le 29 avril 2003, consulté le 20 janvier 2020. URL : http://journals.openedition.org/conflits/829. doi : https://doi.org/10.4000/conflits.829

Certeau, M. de (1990). L'Invention du quotidien. Les arts de faire. Paris : Gallimard.

Chung Van Hoang \& Chong, T. (2017). «Vietnamese Catholics Protests over Environmental Issue: A Test for the State-Church Relationship ». Disponible à : https:// www.iseas.edu.sg/medias/commentaries/item/5457--vietnamese-catholics-protestsover-environmental-issue-a-test-for-the-statechurch-relationship-by-chung-vanhoang-and-terence-chong

Fraser N.(2005). Qu'est-ce que la justice sociale? Reconnaissance et redistribution, trad. de l'anglais par E. Ferrarese. Paris : Éd. La Découverte.

Habermas, J. (1988 [1962]). L'Espace public. Archéologie de la publicité comme dimension constitutive de la société bourgeoise, trad. de l'allemand par M. B. de Launay, Paris : Payot.

Hoang Co Dinh (2017). Formosa - Thảm họa của dân tộc Viêt Nam. CreateSpace Independent Publishing Platform.

Honneth, A. (2013). La Lutte pour la reconnaissance. Coll. Folio essais. Paris : Gallimard.

Lambert, F. (2007). L'Écriture en recherche. Paris : Parcours édition (SIC).

Negt, O. (2007). L'Espace public oppositionnel. Coll. Critique de la politique. Paris : Payot.

Nguyen Thanh Giang (2010). « When Lack of Impartiality Makes an Impact: A Comparative Study of VietCatholic and the BBC ». Reuters Institute for the Study of Journalism, University of Oxford, Oxford.

Nguyen, T.-D. \& Datzberger S. (2018). The Environmental Movement in Vietnam: A New Frontier of Civil Society Activism? Challenging Authoritarianism Series: 4. War and Pacification Project, Amsterdam: Transnational Institute, TNI, 17 p.

Passicousset, L. \& Papin, P. (2010). Vivre avec les Vietnamiens, Paris : Éd. de L’Archipel.

Serres, M. (2019). Relire le relié. Coll. Essais \& Documents. Paris : Éd. Le Pommier.

Souchier, E. \& Jeanneret, Y. (2009). Écrit d'écran. Dans D. Ablali \& D. Ducard (éds), Vocabulaire des études sémiotiques et sémiologiques (pp. 186-187). Coll. Lexica. Mots et Dictionnaires 17. Paris/Besançon : Honoré Champion/Presses universitaires de Franche-Comté. 
Tran Thi Lien, C. (2010). Les relations entre l'Église catholique et l'État au Viêt Nam depuis le Đổi mới. Social Compass 57(3), 345-356. Doi : https://doi. org $/ 10.1177 / 0037768610375519$

Tran Thi Lien, C. (2018). « Le rôle social de l'Église dans un pays socialiste à économie de marché : le cas de l'Église catholique vietnamienne». Mars. Document en ligne : https://www.sciencespo.fr/ceri/fr/oir/le-role-social-de-l-eglise-dans-un-pays-socialiste-economie-de-marche-le-cas-de-l-eglise-catholi

Voirol, O. (2005). Les luttes pour la visibilité. Esquisse d'une problématique. Réseaux 129-130(1-2), 89-121. Doi : https://doi.org/10.3917/res.129.0089.

Voirol, O. (2015). Une critique immanente de la communication sociale. Sur quelques potentiels de l'approche honnéthienne. Réseaux 193(5), 43-77. Doi : https://doi. org/10.3917/res.193.0043 


\section{(c) $(1) \Theta$}

BY NC ND Publié sous la licence Creative Common

«Attibution - pas d'utilisation Commerciale - Pas de Modification 4.0. International» (CC BY-NC-ND) 